\title{
Thermal Unfolding of Antibodies with the Prometheus NT.48
}

Lea Martin, Melanie Maschberger and Dennis Breitsprecher.

NanoTemper Technologies GmbH, Munich, Germany

\section{Introduction}

The development of therapeutic antibodies requires optimal formulations for long-term antibody stability. For this, buffer screening approaches are routinely used, in which the thermal stability of a given antibody in different buffers is tested. The buffers typically vary in buffer composition, $\mathrm{pH}$ values, salt concentrations and other excipients.

The prerequisite for such screening approaches are high measurement precision, low sample consumption, and high throughput. Moreover, it is highly desirable to measure under native conditions, without dilution of the antibody or the requirement to use reporter dyes or other modifications.

Here we present nanoDSF, a label-free, native Differential Scanning Fluorimetry technique, as the method of choice to perform rapid and precise buffer screening projects for therapeutic antibodies during the drug development process.

\section{Results and Discussion}

Our results show that the Prometheus NT.48 delivers highest quality thermal unfolding data for antibody buffer screening campaigns.

The thermal buffer screen of the antibody revealed a strong pH-dependence with an increased stability towards higher $\mathrm{pH}$ values. Future screening campaigns could therefore aim to further investigate thermal stability beyond $\mathrm{pH} 6$.

In addition, the large dynamic range of the Prometheus NT.48 allows for analyzing thermal unfolding in solutions containing antibody concentrations between $200 \mathrm{mg} / \mathrm{ml}$ down to few $\mu \mathrm{g} / \mathrm{ml}$. Thus, it can be utilized for both, stability screening during early phases of antibody development where only small amounts of protein are available, as well as for

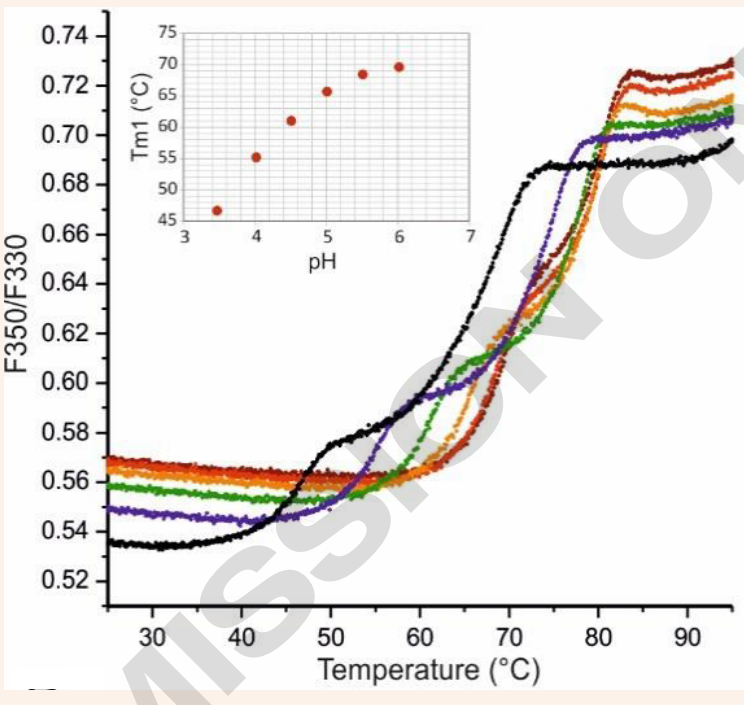

Figure 1. Thermal unfolding curves of a monoclonal antibody in presence of $25 \mathrm{mM} \mathrm{Na}$-Citrate at different $\mathrm{pH}$ values, represented by different colors. The inset shows the $\mathrm{pH}$-dependence of the first unfolding transition midpoint (Tm1).
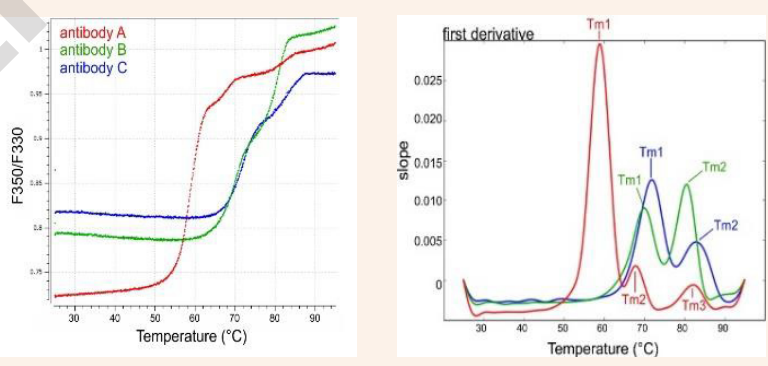

Figure 2. High-resolution data from Prometheus NT.48 thermal unfolding experiments allow for unambiguous discrimination of the unfolding of distinct antibody domains for three different monoclonal antibody samples. Plots of the fluorescence ratio (F350/ final formulation screenings campaigns in highly concentrated samples. F330) and the corresponding first derivative are shown.

Finally, the high-resolution of the thermal unfolding curves is also instrumental for biophysical characterization of antibodies. Thus, the effects of buffers, excipients or even covalent modifications on the stability of distinct antibody domains can be easily assessed

Taken together, due to the high precision and resolution, nanoDSF poses an attractive alternative to orthogonal methods. The Prometheus NT.48 is exceptionally well suited for the rapid and accurate characterization of protein stability even in highly concentrated and viscous samples.

\section{Contact:}

Dr. Heide Marie Resch, Application Specialist \& Marketing

NanoTemper Technologies GmbH

Flößergasse 4, 81369 München, Germany

Phone: +49 (0) 894522895 -25

Email: heidemarie.resch@nanotemper.de

www.nanotemper-technologies.com. 